Koll, Experimentelle Beiträge zur Kenntnis der akuten Quecksilbervergiftung. Dissertation, Würzburg 1897.

v. Kóssa, Zieglers Beitr. z. pathol. Anat., 29. Bd., 1901.

Kunkel, Siłz.-Ber. d. Würzburger physik.-med. Gesellsch. 1889.

Leutert, Fortschritte d. Mediz., 13. Bd., 1895.

Ludwig, Strickers Handb. d. Gewebelehre, 1871.

Marchand, Dieses Archiv, 123. Bd., 1881.

v. Mering, Arch. f. exp. Path., 13. Bd., 1881.

Neuberger, Zieglers Beitr. z. path.' Anat., 6. Bd., 1889.

Ri.cker, Entwurf einer Relationspathologie, Jena 1905.

Rosenbach, Zeitschr. f. rat. Med., 33. Bd., 1868.

Saikowski, Dieses Archiv, 37. Bd., 1866.

Schmidt, Ein Beitrag zur Frage nach der Elimination des Quecksilbers aus dem Körper mit besonderer Berücksichtigung des Speichels. Diss., Dorpat, 1879.

Schweigger-Seidel, Die Nieren des Menschen und der Säugetiere in ihrem feineren Bau. Halle, 1865.

Senger, Langenbecks Arch., 38. Bd., 1889.

Virchow, R., Dieses Archiv, 12. Bd., 1857.

Weichselbaum, Centralbl. f. allg. Pathol. u. pathol. Anat., 2. Bd., 1891.

\title{
XXIV.
}

\section{Über eine Entwicklungsstörung im Kleinhirn in einem Fall von Spina bifida lumbosacralis.}

(Aus dem Pathologischen Institut in Heidelberg.)

Von

M. Gredig.

(Hierzu Tafel XV.)

Vor einigen Jahren hat Ernst zum erstenmal auf die Wichtigkeit einer genauen und systematischen Untersuchung des Zentralnervensystems bei Gehirnbrüchen hingewiesen und diesbezüglich verschiedene wertvolle Befunde zutage gefördert. Angeregt wurde er dazu durch die oft vorgebrachte Meinung, daß vollständige und partielle Defekte des Schädeldaches im allgemeinen und speziell in der Mittellinie des Schädels, also Encephalocelen, im Grunde genommen eine und dieselbe Störung darstellen, nur mit ihren graduellen Besonderheiten versehen. In einer Darstellung der Befunde am Zentralnervensystem eines 
Falles von Encephalomeningocele occipitalis kommt er auf die Beschreibung von Anomalien am Kleinhirn, die er in die Reihe der Aplasien und der Mikrogyrie stellt. Sehr interessant ist der Befund am Wurm und die divertikelartige Ausbuchtung des Hinterhirns zwischen die Hemisphären hinein. Die Vermutung von Ernst, daß eben dieser zwischen die Hemisphären ausgestülpte Teil des Ventrikeldaches ein Rudiment des im frühesten Embrionalleben in seiner Entwicklung gestörten Wurmes sei, verdient, glaube ich, volle Beachtung. Ich meine auch, daß der zweite Fall von Ernst, wo es sich um eine MiBbildung des Kleinhirns beim Erwachsenen handelte, in derselben Richtung wichtig ist. Als ich die Arbeit las, kam mir der Gedanke, ob es sich auch hier vielleicht um eine latente Spaltbildung handelte, vielleicht um eine Spina bifida occulta. Der Verfasser erwähnt aber nichts davon; es fehlen auch Angaben über Begleiterscheinungen einer solchen. Aus diesem Grunde mußte ich meine Vermutung auf sich beruhen lassen.

Nun ist mir in einer Reihe von Fällen, die sämtlich mit Spina bifida verbunden waren, ein Fall begegnet, dessen Befunde am Kleinhirn Erwähnung verdienen.

Es ist ein Fall von Myelomeningocele sacralis posterior, dessen Kleinhirn und Stamm hier einer näheren Untersuchung unterzogen werden soll.

Es handelte sich um ein atrophisches Kind von zehn Tagen. Aus dem Sektionsbefund will ich nur hervorheben, daß am Großhirn beide Occipitallappen besonders deutlich durch Furchen von den Parietallappen abgegrenzt waren; es bestand kein Hydrocephalus.

Das Kleinhirn erschien schon makroskopisch durch eine tiefe Furche an der oberen gegen das Großhirn gerichteten Fläche in die beiden Hemisphären geteilt. Der Monticulus war gar nicht vorhanden. Die Furche setzte sich weiter nach hinten auf die untere Seite fort, ging also allmählich in die Vallecula über, doch war ihre Konfiguration hier nur an der vorderen Hälfte zu erkennen, da die hintere Fläche nach der Medulla oblongata zu von der Pia bedeckt war. Die Lappenteilung des Kleinhirns läßt sich, da die Pia erhalten bleiben soll, nicht genau erkennen. 
Das Kleinhirn wurde mit der Oblongata und einem Teil des Stammes in toto eingebettet.

Nun werden Kleinhirn mit dem Stamm in Serien zerlegt von etwa 25-30 $\mu$ Dicke, und zwar so, daß die Schnittebenen immer senkrecht gegen die Oblongata gerichtet sind, und das Kleinhirn folglich, seiner Lage gegen den Stamm entsprechend, schief getroffen wird. Ich habe fast die ganze Serie, abgesehen von einer Anzahl der am meisten distal gelegenen Schnitte, die nach van Gies on gefärbt sind, ausschließlich nach der Weigertschen Methode behandelt, da sich die Palsche Modifikation und somit auch eine Kernfärbung nicht gut ausführen ließ, wie ein Versuch gezeigt hatte. Das Präparat hat sehr lange in Alkohol gelegen und ist dadurch zu sehr entchromt mir zugekommen.

Die Serie beginnt etwa im unteren Viertel der großen Olive. Der Klarheit und Einheitlichkeit der Darstellung halber sei es mir erlaubt, den ganzen Stamm zunächst und für sich zu besprechen und das Kleinhirn einer daran sich anschließenden Untersuchung zu unterziehen.

Es fällt schon makroskopisch auf, daß die Medulla oblongata bis zum Pons hinauf eine beträchtliche Verkleinerung ihres queren Durehmessers erfahren hat; es macht den Eindruck, als ob das Gebilde von beiden Seiten einem Druek ausgesetzt wäre. Die Hinterstränge treten äußerlich mit ihren Kernen als rundliche Höeker hervor, ebenso die Pyramiden; es besteht aber eine gewisse Asymmetrie, indem die rechte der linken in der Größe etwas nachsteht, ein Umstand, dem übrigens seiner Häufigkeit wegen auch bei ganz normal gebildeten Gehirnen keine besondere Bedeutung zuzuschreiben ist. Dieses Verhalten ist in der ganzen Ausdehnung der Pyramiden zu verfolgen.

Es fällt zunächst mikroskopisch auf, daß an allen Schnitten die Markscheidenbildung sehr schwach ist und anscheinend eine Verzögerung erfahren hat. Es sind nur die Hinterstränge mit ihrer cerebralen Fortsetzung, der Schleife, dann die Vorderstranggrundbündel und ein Teil des Vließes der unteren Oliven, die einigermaßen gut entwickelte Markfasern besitzen. Die meisten Regionen des Querschnittes stellen sich im Weigertpräparat als blasse Bündel und Züge dar. Der Zentralkanal 
weist ein interessantes and jedenfalls nicht zufälliges Verhalten auf. In der Höhe der ersten Zellen des XIT. Nervenpaares stellt er sich als feiner langer medianer Spalt dar; er behält diese Form auch weiterhin, wo er normalerweise als klaffende Fläche am Beginn des IV. Ventrikels sich uns präsentieren sollte. Es scheint mir, daß diese Form einem rein mechanischen Vorgang, welchen ich oben erwähnt habe, nicht allein zugeschrieben werden darf; denn höher, gegen die Mitte der Rautengrube in der Höhe der Brücke, wo sich auch Zeichen eines seitlichen Druckes bemerkbar machen, sehen wir den Zentralkanal, der sich nun zum Boden des Ventrikels ausgebildet hatte, eine flächenhafte Ausdehnung gewinnen, mit einer medianen, spitz zulaufenden Einsenkung, die wohl noch an seine mehr distal gelegene Form erinnern könnte.

Was nun die Architektur der Schnitte betrifft, so sind die Verhältnisse folgende. An einer Anzahl Schnitte, die der Region der unteren Olive entsprechen, finden wir gut entwickelte Hinterstränge mit den Nuclei graciles et cuneati und ihren abgesprengten, mehr der Peripherie zugekehrten Teilen: Nuclei externi von Monakow. Der Keilstrang und sein Kern sind lateral durch ein starkes markhaltiges Bündel, welches wohl zum Hinterstrang zu rechnen ist, von der Substantia gelatinosa und der spinalen V. Wurzel getrennt. Mehr ventralwärts von den genannten Gebilden findet sich der faserarme Kleinhirnseitenstrang mit seinem dorsalen und ventralen Anteil. Die Tractus rubrospinales, spinotectales et thalamici sind nicht sicher voneinander zu trennen. Ventral vom Kleinhirnseitenstrang, in der Gegend der eben genannten Bahnen, finden sich die zerstreuten Nuclei laterales tegmenti, die weiter cerebralwärts an Mächtigkeit zunehmen und schließlich zu einem deutlich abgrenzbaren Zellhaufen etwas medial vom Kleinhirnseitenstrang werden. Schon hier lassen sich in der Substantia reticularis grisea medial und mehr ventral die Anfänge der Nuclei ambigui erkennen; die anfangs zerstreuten Zellen sammeln sich zu einem deutlich sichtbaren Haufen. Lateral von der Schleifenkreuzung und den Vorderstrangresten finden sich bereits die Kerne des Vorderstranges als große Zellen; ebenso findet man die Anfänge des IX., X., XII. Hirnnerven und die 
Nuclei arcuati der äußeren Bogenfasern. Weiter cerebralwärts ändert sich das Bild insofern als an Stelle der mehr kompakten Bündel der Schleife mehr feine, spärliche Faserzüge die Haube durchziehen. In der Medianlinie beginnt sich beiderseits die weiße gitterförmige Substanz in zwei Abschnitte zu sondern; letzteres wird durch den sich einsehiebenden Nucleus reticularis tegmenti bedingt. Seitlich am Außenrande nimmt der Kleinhirnseitenstrang an Masse etwas zu; er schiebt sich weiter nach oben und mischt sich mit der immer breiter werdenden spinalen Wurzel des Trigeminus. Unverändert erscheinen die Substantia gelatinosa, die Tractus spinotectales et rubro-spinales, die Oliven und Pyramiden.

Ein Gebilde verdient aber eine nähere Besprechung. Beiderseits findet sich über den Hypoglossuskernen eine ovale bis runde Zellanhäufung, die ganz wesentlich vom Typus eines motorischen Kernes abweicht. Beide sind von einem Mantel feinster, sich durchflechtender markhaltiger Fasern umgeben. In einem reticulierten Grundgewebe finden sich reichlich mittelgroße blasige Zellen, die durchaus nicht den Charakter multipolarer Ganglienzellen haben. Merkwürdig ist nun der Umstand, daß man an vielen Schnitten beide Gebilde zugleich antrifft, dann verschwindet eins im nächsten Schnitt z. B., um im dritten wieder zum Vorschein zu kommen. Es könnte nun die Frage entstehen, ob wir es hier nicht mit dem Nucleus funiculi teretis, der manchmal eine ansehnliche Größe erreicht, zu tun haben. Dagegen würde weniger die tiefe Lage, als das plötzliche Auftreten in großer Ausdehnung sprechen, dann aber vor allem das eigentümliche Verhalten des Verschwindens und Wiederkommens, wie es hier der Fall ist, und schließlich auch die Größe der ganzen Zellanhäufung. Der N. funiculi teretis stellt sich im Schnitt als ein meist kleiner, nicht scharf begrenzter Zellkomplex dar. Um eine Verwechslung mit den umliegenden Kernen der drei früher genannten Nerven kann es sich nicht handeln, da diese auf den betreffenden Schnitten sich auch ganz deutlich an ihren Stellen vorfinden. Außerdem spricht die Form der Zellen des Gebildes meiner Ansicht nach dagegen. Nun treten aber nach Oberstein er atypische rundliche Zellkomplexe inmitten verschiedener motoriseher Kerne 
auf, besonders soll es der Fall sein beim Hypoglossus; nach der Beschreibung, welche Obersteiner von diesen Gebilden gibt, könnte es sich in meinem Fall darum handeln, doch muß ich hervorheben, daß sich die fraglichen Zellgruppen nicht im Hypoglossus selbst vorfinden, sondern medial von ihm. Professor Obersteiner, der die Güte hatte, seine Ansicht über diese Gebilde in meinem Fall zu äußern, spricht sich entschieden dafür aus, daß es sich auch hier um eine solche atypische Zellgruppe im Hypoglossus handelt.

Medial von der Übertrittsstelle der Fibrae arcuatae in die Nuclei cuneati et graciles findet sich eine rundliche Anhäufung gliöser Substanz von markhaltigen Fasern umgeben, es ist dies die gelatinöse Substanz und die Wurzel des Glossopharyngeus.

Von nun an wird der Zentralkanal klaffend angetroffen, die Brücke, die ihn von der hinteren Längsspalte trennte, verschwindet. Das Lager von Stützsubstanz um den nun zum offenen Spalt und somit zum Beginn der Rautengrube gewordenen Zentralkanal gewinnt sehr an Dicke; schon mit schwacher Vergrößerung erkennt man außer früher angeführten Kernen die Anfänge des kleinzelligen Acusticuskernes. Das runde Gebilde, welches wir in den vorigen Schnitten sahen und beschrieben hatten, ist vollends verschwunden und kommt auch nicht mehr zum Vorschein; auch ein Beweis vielleicht gegen die Annahme, daß es sich um den Nucleus funiculi teretis handelte.

Die Nuclei graciles haben jetzt einem gliösen Maschenwerk mit sehr spärlichen Ganglienzellen Platz gemacht; der Nucleus cuneatus wird mehr zerklüftet angetroffen. Die spinale Trigeminuswurzel mit ihrer gelatinösen Substanz ist mehr dorsomedial gerückt; sie erscheint sehr deutlich als ein längliches viereckiges Feld markhaltiger Fasern. Ihre gelatinöse Substanz verliert ihr gleichmäßiges Aussehen, wird buchtig und durch markhaltige Fasern durchsetzt; in manchen Schnitten findet man abgesprengte Teile nahe dem Nucleus cuneatus, medianwärts daneben finden sich die immer deutlicher hervortretenden Teile der spinalen V. Wurzel Fibrae comitantes. Lateral an der Außenseite schiebt sich successive die Kleinhirnseitenstrangbahn über die V. Wurzel, beide Gebilde zeigen die Tendenz, immer 
mehr dorsalwärts zu gelangen. Die dorsale Hälfte des Kleinhirnseitenstranges umgeht auf diese Weise die spinale V. Wurzel und den $\mathrm{N}$. cuneatus und hilft das Corpus restiforme bilden. In der weißen reticulierten Substanz sondert sich der obere Teil der Fasern zum Fasciculus longitudinalis posterior. Unten, ventral von den Vorderstrangresten, hebt sich deutlich die Schleife ab, quer von den interolivaren Fasern durchzogen. An den letzten Schnitten dieser Reihe bemerkt man unter dem großzelligen Hypoglossuskern im hinteren Längsbündel eingelagert eine rundliche Gruppe von kleinen multipolaren Zellen auftreten, den accessorischen Kern von Roller.

Wir finden weiter cerebralwärts alle früher beschriebenen Gebilde meist ohne wesentliche Veränderungen in Lage und Form. Der Kleinhirnseitenstrang ist nun vollends, ins Corpus restiforme übergegángen, letzteres ist aber noch recht schmal und bandförmig ausgezogen. Was bisher auffiel, ist die fast völlige Abwesenheit der Fibrae cerebello-olivares, die man hier gerade erwarten konnte. Die allmählichen Veränderungen im Bau des Querschnitts, die wir besprochen haben, machen sich nun geltend mit dem völligen Verschwinden der unteren Olive. Unter dem Boden des IV. Ventrikels machen sich die Umrisse des dreieckigen Acusticuskernes immer mehr bemerkbar. In der Substantia reticularis grisea findet man nicht mehr jene geschwungenen quer verlaufenden Fasern. Auch in der Substantia alba sind Veränderungen eingetreten; wir finden das hintere Längsbündel und die mediale Schleife ganz scharf gesondert. Seitlich von der Stelle, die die untere Olive einnahm, treten feine markhaltige Fasern des Corpus trapezoides auf mit den ersten. Zellen der oberen Olive.

In den Schnitten, in denen die Brücke zum Vorschein kommt, sieht man, wie die Pyramidenbahnen, die ursprünglich direkt unter der Haubenregion gelegen waren, nun herabzusteigen anfangen; dies erkennt man an den Schief- und Längsschnitten, die die Bündel zeigen. Etwa um die Mitte der Brücke sieht man die Schiefschnitte verschwinden, die Bahn hat eine mehr horizontrale Richtung eingeschlagen, und es erscheinen nun die Bündel quergetroffen. Die Brücke ist beiderseits symmetrisch gebaut, erscheint sehr arm an mark- 
haltigen Fasern; es sind nur die äußersten, die eine schwache Markscheidenbildung aufweisen; sonst erscheint der ganze Querschnitt in der ganzen Ausdehnung vom distalen bis zum cerebralen Ende blaß mit eingelagerten kleineren und größeren Zellen, offenbar den Brückenkernen und Gliazellen.

Der Teil des Trapezkörpers, der aus dem Nucleus accessorius allmählich mit dessen voller Ausbildung entspringt, wird von den Fasern des N. vestibularis durchflochten und begibt sich an die laterale Seite der oberen Olive. Die Corpora restiformia zeigen nun in gewissen Höhen kleine rundliche Anhäufungen von Zellen; die successive Untersuchung dieser Gebilde zeigt, daß sie in die Tubercula acustica übergehen rnd wohl durch die Fasern des zur Auṣbildung gekommenen Corpus restiforme abgesprengt wurden.

Ähnliche Gebilde, die ich auch in der eben angegebenen Weise deuten möchte, finden sich in der Masse des Hemisphärenmarkes des Kleinhirns, an Stellen, die unmittelbar dem Stamme anliegen; ein Umstand wird wohl diese Verlagerung begünstigt haben, nämlich der, daß die seitlichen Ausbuchtungen der Ventrikelhöhle nicht allzu tief reichen und einem solchen Übertreten der Zellmassen nicht im Wege standen.

Gegen die Mitte des Pons sieht man, wie das Acusticussystem, das schon früher seine volle Entwicklung erreicht hat, allmählich an Elementen verliert; es verschwinden zunächst die Verbindungen des Tuberculum acusticum mit dem Stamm des Nervus cochlearis. Das Corpus restiforme geht nun in die Faserung des Kleinhirns über; aus seinen oberen und unteren lateralen Partien zweigen sich feine Bündel ab und gehen in einem nach außen konvexen Bogen um den Nucleus dentatus herum nach oben, um über der Kuppe des gezackten Kernes zu verschwinden; vereinzelte Fasern kann man bis nahe an den oberen Wurm herantreten sehen. Der Strickkörper sowie die Substantia gelatinosa mit ihrer Wurzel sind stark lateralwärts gerückt und befinden sich, sehr an Masse reduziert, nahe am Kleinhirn.

Indem sich der laterale Teil des Corpus restiforme von außen am Corpus dentatum anlegt, verlaufen die medialen Teile des Strickkörpers gestreckt an der medialen Seite des 
Corpus dentatum vorbei, zwischen letzterem und dem Wurme nach oben, wo sie sich mit dem äußeren Anteil durchflechten; ein Teil dieser medialen Fasern scheint aber auch gegen den unteren Wurm hinzuziehen. In dem Maße, als sich der Strickkörper auflöst, bemerkt man nach innen von ihm, durch seinen medialen Teil bedeckt, die quer- und schiefgetroffenen Bündel der Bindearme, die eine Richtung von oben nach unten und vorne einschlagen. Unter dem Bindearme sieht man alle Komponenten des Trigeminus zum Vorschein kommen; die cerebrale Wurzel, darunter die sensiblen und motorischen Kerne, mit ihren Wurzeln und mehr nach unten den Schiefschnitt des gemeinsamen Stammes.

Nun sind wir in der Durchsicht jenseits auf die cerebrale Brückenhälfte angelangt. Das Acusticussystem ist fast gänzlich verschwunden. Wir treffen noch auf wenige Züge des Trapezkörpers, die sich scharf durch die längsgetroffenen Fasern von den in der Längsrichtung des Stammes verlaufenden Bündeln der medialen Schleife abheben. Die laterale Schleife, die sich hier zu sammeln anfängt, drängt den Bindearm allmählich gegen die Medianlinie zu; ihre Fibrae perforantes, die sich abzweigen und den unteren Teil des Bindearmes durchziehen, verleihen diesem ein zerrissenes Aussehen. Die weiteren Verhältnisse am Stamm in den mehr cerebral gelegenen Partien ergaben bei einer sorgfältigen Durchmusterung der Serie nichts, was ein besonderes Interesse beanspruchen könnte. Deswegen will ich ihre weitere Beschreibung unterlassen. Es ergaben sich also nur wenige, meist geringgradige Abnormitäten im ganzen Stamme, die in dem Vorgebrachten gänzlich erschöpft wurden.

Bevor ich zur Besprechung des Kleinhirns, speziell des Wurmes übergehe, möchte ich noch zunächst des hinteren Marksegels Erwähnung tun.

Wir sahen, wie der Zentralkanal viel später zur Rautengrube sich entfaltet, als es wohl der Norm entsprechen würde. In einer ganzen Reihe von Schnitten erscheint das Dach des IV. Ventrikels in seiner ganzen Ausdehnung stark seitwärts und nach unten ausgeweitet. Dabei sieht man, wie die Tela chorioides, die normalweise über der Rautengrube gespannt 
sein sollte, in den distalen Schnitten bei ihrem Hineinwachsen das Dach nach den Seiten und nach unten ausdehnt. Weiter cerebralwärts, wo die Tela ins Velum medullare übergeht, findet man dieses auch noch in derselben Weise ausgedehnt; man erkennt dies sofort am Ventrikelependym, das den feinsten Ausstülpungen und Aussackungen in der Konfiguration des Ventrikels folgt.

Ein Hydrops, der eine Erweiterung der Kentrikelhöhle bedingt hätte, war in unserem Falle nicht vorhanden; wir müssen es also wohl mit einem Zustand zu tun haben, der schon von vornherein angelegt sein mußte. Es macht den Eindruck, als hätten wir es hier mit einem Stadium der Entwicklung zu tun, wie es viel früher in den ersten Wochen des Embryonallebens normalerweise der Fall ist, wo dieser Abschnitt des Nervensystems einen noch relativ sehr großen Hohlraum besitzt. Nun kann man annehmen, daß, indem das Gehirn sich normalerweise weiter entwickelte, die oberen Partien ihren ursprünglichen Charakter beibehielten; es würde sich hier gewissermaßen um eine Hemmungsbildung im Dach des Ventrikels handeln. In einer Höhe, die etwa der Mitte des Pons entsprechen würde, verschwinden die seitlichen Taschen des Ventrikels; das Lumen erscheint jetzt in der Richtung von oben nach unten durch die stark vorspringende Lingula fast verlegt, um später nach dem Verschwinden der letzteren in den Aquaeductus Sylvii überzugehen.

Ich will nun aus der etwas langen Besprechung des Stammes seine wenigen Abnormitäten der Übersicht wegen kurz zusammenfassen.

Aufgefallen ist mir vor allem der kurze seitliche Durchmesser des Stammes und das starke Hervortreten der Hinterstränge, dann die ausgezogene Form des Zentralkanals, der hier schon in der Höhe der unteren Olive die bekannte rundliche Form des Anfanges der Rautengrube zeigen sollte. In dem Bau der Querschnitte hatten wir eine allgemeine Verzögerung -der Markscheidenausbildung zu verzeichnen. Dann sahen wir, wie beiderseits rom Zentralkanal ein Gebilde auftrat, dessen sonderbares Verhalten ich seinerzeit besprochen habe. Weiter cerebralwärts von dem Beginn der Brücke fanden wir 
den Kleinhirnseitenstrang noch recht schmal und das Corpus restiforme wenig vorspringend, wohl beides durch den Markscheidenmangel bedingt; hier ist uns anch der fast vollständige Mangel der Oliven-Kleinhirnbündel aufgefallen. In der Höhe des Acusticus sahen wir seitlich am Stamm sehr stark entwickelte Tubercula acustica; an Stellen, wo der Stamm im unmittelbaren Zusammenhang mit den Kleinhirnhemisphären stand, konnte man nachweisen, wie die Zellen' dieser Kerne auch in die anstoßenden Teile des Kleinhirns übergriffen, es wäre somit auch eine mächtige Entwicklung dieser Teile des Acusticus anzunehmen. Endlich haben wir die angeführten Verhältnisse am hinteren Marksegel zu erwähnen.

Die Kleinhirnhemisphären kamen nicht in gleicher Höhe in den Schnitt. Man könnte versucht sein, bei der Durchsicht einzelner Schnitte des Objekts an eine Asymmetrie zu denken. Eine systematische Untersuchung der Hemisphären ergab, daß sämtliche Teile der rechten Seite in Schnitt und Größe denjenigen der linken nachstanden, außerdem konnte ich aber na.chweisen, daß sich diese Verhältnisse successive ausglichen, indem die Gebilde der rechten Seite nach und nach zur selben Größe, wie es auf der linken war, heranwúchsen. Deshalb kann es sich hier nicht um eine ungleichmäßige Entwicklung handeln, sondern lediglich um eine Verschiebung der Hemisphären gegeneinander. In der weißen Substanz macht sich dieselbe Verzögerung der Markbildung kund wie am Stamm. Die accessorischen Kerne - Nuclei globosus - und emboliformis kommen viel früher zum Vorschein als die Corpora dentata, fallen aber sofort auf; dagegen hatte ich gewisse Schwierigkeiten, mich der Anwesenheit des Dachkernes zu vergewissern, da er durch den mißbildeten Wurm verzogen und durch unregelmäßige markhaltige Züge zerklüftet war.

Direkt an den Stamm legt sich beiderseits ein mächtig: entwickeltes Adergeflecht; rechts kommt es nach und nach zur Entwicklung und stößt mit dem anderseitigen an der dorsalen Seite zusammen. Schon sehr bald nachdem die Hemisphären des Kleinhirns sichtbar werden, schiebt sich zwischen sie eine Anzahl quergestellter Windungen von oben her; gleichzeitig aber kommt zwischen den erwähnten Adergeflechten in 
der Medianlinie und unter den querverlaufenden Windungen, die unschwer als die Anfänge des Wurmes zu erkennen sind, ein zapfenförmiger Fortsatz, von einem Bau, der anscheinend Ähnlichkeit mit Nervensubstanz besitzt; er liegt zunächst ganz isoliert da und ist von den beiden Adergeflechten umschlossen, später aber geht er nähere Beziehungen mit den quergestellten Windungen ein. Es werden die oberen Windungen von dem unteren Zapfen durch Piagewebe mit reichlichen Gefäßen, dann gliösen Massen voneinander getrennt. Je höher man im Schnitt gelangt, desto schmäler wird die Piamasse und desto näher rücken beide Teile aneinander, bis sie schließlich verschmelzen. Die erwähnten queren Windungen und unteren Gebilde sind zunächst in keinem nachweisbaren Zusammenhang mit den Hemisphären; sie sind ringsherum von Piagewebe umgeben und behalten ihre Selbständigkeit eine Zeitlang, bis sie mit den Windungen der Hemisphären zusammenfließen. Auf diese Weise ist es schon auf den ersten Blick klar, daß wir es hier mit dem Wurme in seiner ganzen Ausdehnung zu tun haben, und zwar mit der hinteren etwas frei zwischen die Hemisphären vorragenden Partie. Der Zapfen wird weiter cerebralwärts immer breiter, stumpfer und nimmt in einer gewissen Höhe, die etwa dem vorderen Abschnitt der unteren Olive entsprechen würde, die ganze Breite des Wurmes ein. Der obere Teil des Wurmes erreicht die Ränder der Hemisphären nicht; es bleibt eine seichte Einsenkung bestehen, die ich für den Schnitt durch die Vallecula halte; wir hätten somit den unteren Wurm vor uns. Etwa zu drei Vierteln besteht er aus ganz ordnungsmäßig gestellten Windungen, die sich durch nichts in ihrem Bau von einer normalen Rinde eines Nengeborenen unterscheiden. Nach unten zu wird der übrige Teil durch den Zapfen eingenommen und zwischen beiden liegt ein ziemlich breites Lager von Markfasern, das ich für den Querschnitt des horizontalen Astes des Markkernes halte. In dieser Höhe sollte nun am normalen Präparat das Knötchen die Stelle des Zapfen einnehmen. Da es mir bis jetzt nicht gelungen ist, eine Marke herauszufinden, die irgendeine Teilung des Zapfens ermöglichte, halte ich ihn für die Anlage der nichtdifferenzierten Uvula und Nodulus des unteren Wurmes. 
Der Zapfen vergrößert sich ganz allmählich und zeigt gewisse Beziehungen zum Boden der Rautengrube. Sein unterer Rand erscheint stellenweise sofort an den Boden des Ventrikels angepreßt, daß es wohl den Anschein erwecken könnte, als wenn beide miteinander verwachsen wären. Bei genauerem Zusehen, kann man indessen deutlich an der Berührungsstelle das Ependym des Ventrikels nachweisen (Fig. 1, Taf. XV). Es erscheint oftmals gelockert, die Abstände seiner Zellen scheinen größer geworden zu sein und die Kerne sind nicht so deutlich wie sonst sichtbar; an manchen Stellen fehlt das Epithel gänzlich und der Rand des Gebildes ist aufgefasert. Endlich konnte ich Stellen finden, wo es den Anschein machte, als ob die Substanz des Wurmes in die des Bodens des Ventrikels direkt überginge. Bei stärkerer VergröBerung konnte man aber hie und da Reste von Ependymepithel als Grenze nachweisen; es sah so aus, als ob die Zellen beider Flächen aneinander geschoben wären.

Eine Reihe von Schnitten zeigt, daß der Zapfen in seiner Größe gegen das Gehirn zu abnimmt, er wird immer schmäler und unregelmäßiger in seiner Form, bis schließlich seine letzten Rudimente, mit Einstülpungen des Ependyms versehen, sich ausgleichen und einer etwas gegen das Ventrikellumen zu konvexen Fläche Platz machen. Dies ist die Stelle, wo auch im normalen Präparat das Knötchen allmählich verschwindet und der flachen unter dem Dachkerne befindlichen Kuppe des Ventrikels Platz macht.

Die feinere Untersuchung zeigt, daß der untere Teil des Wurmes eine Architektur besitzt, die ganz wesentlich von derjenigen des übrigen bisher betrachteten Teiles des Kleinhirns abweicht. Wie schon betont wurde, liegt zwischen beiden Teilen des Wurmes ein starker Markkegel. Darunter beginnt der ganzen Breite nach eine regelrechte Schicht von Körnern, begrenzt nach unten von einer Reihe ziemlich dicht gestellter Purkinjescher Zellen. Wenn wir einen Blick auf den oberen Teil des Wurmes werfen oder auf eine beliebige Stelle der Hemisphärenrinde, so sehen wir folgendes Verhalten: eine schmale äußere Körnerschicht, in der man stellenweise auch noch die Sonderung in eine äußere dichtere Zone mit dicht aneinander gedrängten Körnern und eine immer mehr locker gefügte Schicht unter- 
scheidet. Dann folgt die ziemlieh breite Molekularlage, welche an den Präparaten trotz der nicht geringen Dicke des Schnittes von $25 \mu$ sehr deutlich ihre radiäre Strichelung erkennen läbt; man kann hier außer versprengten Zellen sehr feine punktförmige Zeilen erkennen - offenbar sind es Querschnitte der markhaltigen Fortsätze der Körner aus der darunter liegenden Schicht, die sich in der Molekularlage in verschiedenen Höhen verteilen. Es folgt nun die sehr deutliche Ganglienzellenschicht und dann die Körner mit den Markstrahlen.

Die Purkinje-Zellen kann man an beliebigen normalen Stellen stets in einer Reihe gelegen antreffen, den Dendriten gegen die Molekularlage gerichtet und annähernd parallel gestellt.

In dem in Frage stehenden Teil des Wurmes ist nun die Anordnung der Zellen so, daß sie aus ihren Reihen austreten, mehr oder weniger in die folgenden Teile sich einschieben und ihre Fortsätze nach allen Richtungen senden, also die typische Verteilung nicht aufweisen. Unter diesen Reihen, die gewissermaßen den Ubergang bilden, folgen Bezirke, wo man keine einheitlichen Schichten erkennt. Von allen Seiten umgeben dichtgedrängte körnige Elemente große blasige Zellen and setzen sich fast ebenso dicht gestellt weiter nach unten fort, schichtenweise, in mehr oder weniger langen Absätzen durch Stränge und Streifen von einer Substanz, die große Ähnlichkeit mit der Molekularschicht der Windungen hat, in größere und kleinere Haufen zerlegt. Dazwischen liegen nun überall regellos zu Haufen gruppiert große Zellen, an denen man oft den charakteristisch verzweigten Dendriten der Purkinje-Zelle erkennen kann. Dieses eben geschilderte Verhalten betrifft die ganze untere zapfenförmige Masse. Die Teile sind in ein Maschenwerk gliöser Substanz eingelagert, und es weist der Zapfen mehrere solche Bezirke auf. Die Mitte derselben hat ein lockeres Gefüge; es bilden sich Spalten zwischen einzelnen Zellen und Zellkomplexen, die hie und da keinen regelmäßigen Verlauf haben, oft aber parallel zu einandergehen. Es machen diese Bezirke den Eindruck, wie Ernst es in bezug auf seinen Fall sagte, als ob hier die nervösen Elemente einer Rinde durcheinander gewürfelt und gemischt wären. Von markhaltigen Fasern habe ich nur Spuren auffinden können und meistens 
am Rande; was aber bemerkenswert ist, das ist die überaus starke Vascularisation des ganzen Gebildes.

Gegen die untere Peripherie des Zapfens, und in dem Maße, als er seinem Ende sich naht, nehmen die nervösen Elemente zusehends an Zahl ab. Die Purkinje-Zellen verschwinden, dann auch die Körner und in den letzten Schnitten, wo das Gebilde noch zum Vorschein kommt, findet sich nur noch ein Gliareticulum.

Wir sahen, daß zwischen den beiden Teilen des Wurmes sich allmählich ein Markkegel zeigte und eine Grenze bildete. Nun wird der Markkern noch in der Höhe der größten Ausbildung des Zapfens sichtbar. Inmitten des Markkerns taucht ein kreisrunder Fleck auf; wie man sich leicht an aufeinanderfolgenden Schnitten überzeugen kann, besteht er aus grauer Substanz. Es treten auf einmal eine Menge runder Zellen auf von gleicher Größe und gleicher Dichtigkeit in der Verteilung; soweit die genauere Untersuchung dieser Zellen möglich war, stimmten ihre Formen mit denjenigen der Körner überein; dann außer diesen fanden sich zerstreut große blasige Gebilde, bei denen man oft den Anfang eines dicken Fortsatzes unterscheiden lkonnte, es kamen auch Stellen vor, wo ich direkt den Baum der Purkinje-Zellen an ihnen nachweisen konnte (Fig. 2, Taf. XV). Nach einem Vergleich mit Elementen einer normalen Rinde kam ich zu dem Sehluß, daß ich es hier mit genau denselben Gebilden zu tun habe.

Das eben Ausgeführte beweist die Zugehörigkeit des Fleckes zur grauen Substanz und zwar zur Rindensubstanz. Wenn ich hier von einer Zugehörigkeit zur Rindensubstanz rede, will ich betonen, daß das Gebilde aus den Elementen der Rinde zusammengesetzt ist; damit ist aber vorläufig die Ubereinstimmung erschöpft.

Für die Rinde sind nicht nur bestimmte Elemente typisch, sondern auch ihre Lage zueinander, ihre Architektur; nun ist aber an der vorliegenden Stelle von einer Architektur gar nichts zu finden. Der Zellhaufen vergrößert sich rasch, dabei bleibt er immer von einem ziemlich dichten Markmantel umgeben; die Richtung der Fasern darin ist keine einheitliche, sie verlaufen vielmehr nach allen Seiten und man erkennt oft, wie 
viele von ihnen einzeln und in Bündel geordnet, tief in die Substanz des atypischen Fleckes - so lrönnen wir ihn wohl jetzt nennen - eindringen und ihn in Felder teilen.

Von einer bestimmten Höhe an macht sich etwas ganz Bemerkenswertes geltend. Es treten an manchen Stellen eines jeden Schnittes in der Gegend der Mitte des Wurmes die Körner in größeren kontinuierlichen und distinkten Haufen von gleichmäßiger Dichte zusammen. Nun kommen in einer folgenden Reihe von Schnitten in diesen eben erwähnten Inseln hellere Stellen auf, die in den nächsten dann eine Anzahl Purkinjescher Zellen enthalten; diese letzteren treten weiterhin mehr an die Peripherie des helleren Zentrums und stellen sich so, daß ihre Dendriten gegen die Mitte gerichtet sind. Auf diese Weise bekommt man folgendes Bild: Wir haben einen ziemlich scharf begrenzten Bezirk von Körnern, dann folgt in der Mitte ein ovaler Ring von locker gewebter Grundsubstanz mit spärlichen Zellen und gleichmäßig verteilten Purkinje-Zellen. Die Masse aller Dendriten ist in ein Gewebe eingelagert, das bei schwacher Vergrößerung dunkler als die anderen Stellen und mehr homogen erscheint. Bei stärkerer erkennt man in ihr eine parallele Querstreifung mit größeren und kleineren Zellen; die Querstreifung wird teils durch die genannten Dendriten, teils durch feinste Gefäße, die in derselben Richtung ziehen, hervorgebracht. Man erkennt daraus, daß wir die Molekularschicht vor uns haben. Nach einer weiteren Reihe von Schnitten tritt inmitten der Molekularlage ein Spalt auf, der diesen Bildungsprozeß beschließt. Wir haben auf diese Weise aus einem ganz verworrenen Konglomerat von Zellen eine ganz regelrecht gebaute Windung entstehen sehen.

Dieser Befund tritt nun, nachdem er zuerst an wenigen Stellen begonnen hatte, successive im oberen Teil des Wurmes auf, immer größere Flächen desselben ergreifend und von oben nach unten fortschreitend. Sehr interessant und wichtig für die Beurteilung der Provenienz speziell der Bruchstücke, die Ähnlichkeit mit Molekularsubstanz hatten, ist, daß an Stellen, wo sich typische Windungen herausbilden, man schon mit schwacher Vergrößerung nachweisen kann, wie diese Züge und Bruchteile auch tatsächlich in die Molekularsehicht der sich eben 
gebildeten typischen Windungen übergehen (Fig. 3, Taf. XV). Somit ist die Zugehörigkeit dieser Züge zur Molekularsehicht erwiesen. Dieses Übergehen in typische Molekularschicht ist nicht etwa an einer Stelle zu treffen, sondern überall, wo sich eine Windung bildet, und deswegen etwas ganz Gesetzmäßiges. Etwa um die Mitte der Rautengrube stellt der Wurm ein ganz charakteristisches Bild dar. Die obere Hälfte besteht aus wohlausgebildeten querverlaufenden Windungen, in die sich feine Markstrahlen einsenken, unten haben wir das Bild der Atypie vor uns; beide Teile sind scharf gegeneinander abgegrenzt. Indem sich von oben her immer neue Windungen herausdifferenzieren, wird der untere Teil kleiner. In der Höhe des Acusticusaustrittes findet man ein Bild, wo nach oben etwa zu zwei Dritteln sich Windungen vorfinden, nach unten davon schon die Querschnitte der Lingula. Weiterhin treten Veränderungen ein, die das Verschwinden des Wurmes einleiten. Es schiebt sich von oben her ein Spalt zwischen die beiden Hemisphären des Kleinhirns, und in dem Maße, als er sich vertieft, nimmt die Höhe des Wurmes ab. Diesen Vorgang kann man bis zu einer Stelle verfolgen, wo der Spalt zwischen den Hemisphären nur noch durch die letzten Windungen der Lingula von der Rautengrube getrennt ist und der Wurm selbst nicht mehr zu sehen ist.

Wenn wir uns Schnitte ansehen, in welchen der früher besprochene untere Zapfen und der atypische Einschluß zugleich vorkommen, so ist bei schon sehr schwacher Vergröberung die ausgesprochene Ähnlichkeit beider Gebilde nicht zu verkennen. Die Regellosigkeit in der Verteilung der Elemente, was beiden gemeinsam ist, die zerklüftete, stellenweise netzförmige Anordnung und das durcheinandergewürfelte Aussehen, sind nur in diesem Sinne zu deuten (Fig. 4, Taf. XV). Die Feinheiten des Bildes sind geeignet, diese Übereinstimmung nur zu erhöhen. In beiden finden wir diese regellose Verteilung so daß die Ganglienzelle inmitten von Fasern steckt, PurkinjeZellen in einem Haufen von Körnern vergraben liegen; in beiden finden wir den enormen Reichtum an Gefäßen, die Unmasse von Spalten zwischen den einzelnen Fragmenten, dann der Charakter der Elemente selbst z. B. der großen blasigen Zellen 
mit mehr oder minder deutlich sichtbaren Fortsätzen - alles dies reiht beide Bildungen in die Gruppe der Atypien und läßt ihre Verwandtschaft außer Zweifel.

Was ist nun das Ergebnis unserer Untersuchung am Wurm? Schon in den ersten Schnitten sahen wir den unteren Wurm als geteiltes Gebilde zutage treten. Seine oberen Partien unterschieden sich durch nichts von den gut gebauten Hemisphären. Der untere Teil, aus der Uvula und dem Knötchen zusammengesetzt, stellte in seinem Bau die oben beschriebenen Unregelmäßigkeiten dar. Bis etwa zur Mitte der Ventrikelhöhle konnten wir diese Unregelmäßigkeiten verfolgen; dann sahen wir, wie die mißbildeten Uvula und Knötchen einem flachen Dache Platz machten. In einer Höhe, wo wir die eben genannten Gebilde noch recht groß sahen, trat im Markkern des Wurmes die besprochene Atypie auf; wir konnten ihre Entwicklung, ihren Übergang in typische Windungen und ihr Verschwinden nacheinander verfolgen. Endlich sahen wir, wie sich der Spalt zwischen die Hemisphären einschob. Die Windungen, die ihn bildeten, gingen in die Hemisphären über, gehörten ihnen also an.

Dieser Spalt war es auch, der schon bei der makroskopischen Untersuchung des Präparats auffiel und einen Wurmdefekt vermuten ließ. Nun, diese Vermutung hat sich auch bestätigt. Von Rechts wegen sollte überhaupt an der Oberfläche des Kleinhirns kein Spalt da sein; an jedem normalen Präparat trifft man hier den Monticulus des Oberwurmes. Also auf Kosten dieses letzteren ist diese Aplasie entstanden; ihre Ursache aber haben wir wohl in der Atypie zu suchen.

Die Störungen, die wir in meinem Fall nacheinander besprochen haben, betrafen den Wurm, den hinteren Teil des Ventrikeldaches und einige Gebilde, die in seiner Nachbarschaft sich befanden. Die Hauptmasse des Stammes ergab so gut wie keine Abweichungen von der Norm. Der Mangel an markhaltigen Fasern, der sich überall kundgab, weist jedenfalls auf eine Hemmung in der Entwicklung der betreffenden Bahnen hin, doch ist er nicht beweisend für ihre Abwesenheit. Ich habe sogar allen Grund zur Annahme, daß sie vorhanden sein mußten, da die zugehörigen Kerne - ich spreche hier über Bahnen, die in Beziehung zum Kleinhirn und speziell zum 
Wurm stehen - in ihrer Ausbildung wenig Auffälliges zeigten. Eine Beeinträchtigung der Funktion (ich spreche dies natürlich nicht nur in bezug auf diesen Fall) des Kleinhirns, namentlich des Wurmes, wäre wohl demnach mit Vorsicht anzunehmen, da, wie wir gesehen haben, gerade die Fasern, die dahin ziehen und von da entspringen, die höchste Stufe der Ausbildung in meinem Falle zeigten. Als Beweis für die Annahme, dab trotz grober Störungen dieses Organ ganz gut seinen Funktionen nachkommen kann, erinnere ich an das mißbildete Kleinhirn eines Erwachsenen im Ernstschen Fall, wo das Individuum sein ganzes Leben ohne wesentliche Anzeichen dieser Abnormität verlebt hatte. Es wäre wohl, nach meinem Fall zu urteilen, nicht ausgeschlossen, daß sich ein ähnlicher Befund am Kleinhirn mancher Fälle von Spina bifida vorfinden würde; auch in solchen vielleicht, die geheilt worden sind, und daß sich dies im Leben nicht dureh irgend welche Störungen zu äußern brauchte.

Ich fand nur in den Fällen von Ernst Analoga zu meinem. Speziell in dem mit Encephalocele fanden sich die Störungen, die mich hier interessieren, in einer ganz scharf umgrenzten Region, am Occiput und den in seiner Nähe sich befindenden Hirnteilen. Die Asymmetrie der beiden Hälften des Stammes, sowie die Verzögerung der Markbildung und Verzerrung der Stammganglien deutete Ernst durch den Druck und Zug des Hydrocephalus (auf die Störungen in der Entwicklung des linken Schläfenlappens gehe ich nicht ein). Über die Beziehungen des Spaltes zwischen den Kleinhirnhemisphären, der Aplasie des Wurmes zum Hydrocephalus, spricht sich Ernst dahin aus, daß diese Störungen als passive Druckwirkungen desselben wohl ohne weiteres aufzufassen wären; er meint ferner, daß es auch denkbar wäre, die heterotopen und atypischen Bezirke in den Hemisphären in Zusammenhang mit den dorsalen Spalten, besonders der Kleinhirnspalte zu bringen. Denkbar ist natürlich, daß ein Hydrocephalus, der auch den vierten Ventrikel betrifft und in einer sehr frühen Periode des Foetallebens eingesetzt hat, das Dach desselben ausdehnen kann und, wenn er vor der Entwicklung des Wurmes begonnen hat, wie es hier dann wohl der Fall sein müßte, auch den Wurm in seiner 
Entwicklung stören könnte. Nun aber ist nicht ganz verständlich, wie sich die Heterotopien aus Mangel an Halt in den Hemisphären entwickeln könnten. Diese letztere Vermutung in der Entstehungsart der Heterotopie stellt Ernst auch für seinen Fall beim Erwachsenen auf. In sehr vielen Punkten ähnelt mein Fall dem Ernstschen. Ein Hydrocephalus war in meinem nicht da, auch keine Encephalocele, aber eine ihr ganz analoge Abnormität, die Spina bifida. Die Höhle des vierten Ventrikels enthielt keine Flüssigkeit; das Dach war stark ausgedehnt, aber nicht nach oben, sondern nach den Seiten des Stammes zu. Diese Ausdehnung konnte also durch keinen Hydrops entstehen, denn gesetzt, es wäre mal früher einer dagewesen und später verschwunden, so hätten sich doch die gedehnten Blätter des Daches bis zur Geburt rückbilden können - eine Tatsache, für die man überall strikte Beweise hat - besonders noch, wo es sich um einen wachsenden Organismus handelt. Der Wurm war hier vorhanden, aber erheblich milggestaltet. Er war ganz der Norm gemäß den Hemisphären angefügt, folglich hatten diese wie auch er selbst genügend Halt aneinander. Trotzdem zeigt er einen atypischen und heterotopen Bau. Die Atypien zeigen die regellose Verteilung der drei Schichten, wie es Ernst für seine Fälle angibt. Stücke von Molekularsehicht mit anliegenden Purkinje-Zellen, deren Fortsätze nach allen Richtungen hinstreben und deswegen von allen Seiten uns zu Gesicht kommen. Wie dort gehen auch hier die atypischen Bezirke allmählich in normal gebaute Windungen über; ich konnte auch bei meinen Schnitten direkt nachweisen, daß das, was mir als Molekularschicht imponierte, auch solche war. Es fehlte in meinem Fall die Verkalkung der Purkinje-Zellen, das wäre aber wohl von untergeordneter Bedentung für die Beurteilung.

Aus eben diesen Gründen meine ich, daß die Vermutung von Ernst über einen Zusammenhang der dorsalen Spalte und der Heterotopie auf Schwierigkeiten stößt. Es wäre nun interessant, etwas über die Entstehungszeit der Mißbildung zu erfahren. Die früheste Periode einer Bildungsanomalie zu bestimmen, ist bei den meisten Bildungsfehlern nicht möglich; der Grund davon ist der, daß eine Ursache, die schließlich zur 
Mißbildung führt, schon sehr früh vorhanden sein kann; sie braucht sich aber nicht sofort zu dokumentieren. Diese Ursache kann zunächst noch auf die weitere Entwicklung ohne merklichen Einfluß sein; wir würden also in diesem Stadium auch bei der genauesten Untersuchung des Objekts nichts Abnormes finden können, obwohl der Keim einer später hervortretenden Anomalie schon angelegt wäre.

Dagegen sind wir imstande, aber auch nur annähernd, den spätesten Zeitpunkt zu bestimmen, die teratogenetische Terminationsperiode, wie sie Schwalbe präzisierte.

Wir fanden in der Atypie Körnerschicht, Molekularsubstanz und Purkinje-Zellen. Gegen das Ende des dritten Monats bekommt, wie bekannt, der Wurm, der sich früher bildet, seine Windungen. Nun haben wir im oberen Wurm oberfächliche Windungen, die nach innen gegen den Markkern zu mit der Atypie zusammenhingen; es würde somit hier die Störung nicht später, als in der Periode der Differenzierung der Windungen zu verlegen sein. Nun aber wissen wir, daß die Scheidung in die drei Schichten am die Mitte der Embryonalzeit geschieht; es hat also den Anschein, als ob unsere eben angeführte Periode zu früh gegriffen wäre. Dem ist aber nicht so, aus dem Grunde, weil die Ursache schon einen geeigneten Boden zur Entstehung der Mißhildung vorbereiten mußte, damit sich später eben die regellose Anordnung in der Verteilung der Sohichten ausbilden könnte; man kann sich sonst nicht denken, warum sich bei der Differenzierung die Molekularschicht eben in der regellosen Weise gelagert hätte und nicht ordnungsgemäß, wie an jeder beliebigen normalen Stelle. Dann haben wir noch die Purkinje-Zellen. Wie soll man sich diese in die Atypie hineindenken, da die Störung, wie wir annahmen, zwischen dem dritten und vierten Monat spätestens einsetzen müßte? Nun, den Purkinje-Zellen erging es ebenso wie der Molekularsubstanz; dies zeigen die kreuz und quer nach allen Richtungen verlaufenden Fortsätze; sie mußten sich eben, gerade wie die Molekularsehicht, den zu dieser Zeit schon gegebenen Verhältnissen anpassen.

lch füge meinen Fall den Ernstschen Fällen an. Er wäre aber vielleicht auch nach einer anderen Richtung von Beden- 
tung - nämlich, da er mit Spina bifida verbunden war - in der Frage des Zustandekommens diesex letzten Mißbildung. Ein wichtiger Angriffspunkt, um der Frage näher zu treten, wäre vielleicht eben das Kleinhirn und der Stamm, da sie ja Störungen aufweisen, die sich an die Medianlinie halten.

Ich möchte noch hervorheben, daß eine genaue mikroskopische Untersuchung auf Serien noch manche Anomalien aufdecken könnte, die makroskopisch kaum anzunehmen sind.

Ein Literaturverzeichnis füge ich meiner Arbeit nicht an; ich lasse dies für eine spätere Arbeit, von der die vorliegende ein Teil ist.

Ich will damit schließen und spreche Herrn Geheimrat Arnold und Herrn Professor E. Schwalbe, deren Ratschläge mir jederzeit zu Gebote standen, meinen tiefsten Dank aus.

\section{Erklärung der Abbildungen auf Taf. XV.}

F i g. 1. Übersichtsbild. Links sieht man einen Teil der Medulla oblongata in der Höhe der unteren Olive; rechts einen Teil der Kleinhirnhemisphären; oben über der Medulla einen Teil des in den IV.Ventrikel hineinragenden Zapfens. a) Untere Olive, b) Kleinhirn, c) Zapfen atypisch gebauter Kleinhirnsubstanz, der Medulla oblongata aufgelagert, d) Ependym des IV. Ventrikels nach der Seite zwischen Kleinhim und Oblongata abnorm stark ausgebuchtet, e) Plexus chorioides des IV. Ventrikels.

Fig. 2. Sehr stark vergröBerte Purkinje-Zelle aus einem atypisch gebauten Bezirk des unteren Wurmes. Die Zelle ist von Molekularsubstanz umgeben, daneben finden sich verstreute Teile der Körnerschicht.

Fig. 3. Schwach vergrößerter Teil des Wurmes. Bei aa) normal gebaute Kleinhirnrinde, bb) atypischer Teil aus durcheinandergeworfenen Teilen der Kleinhirnrinde bestehend. Bei cc) Ubergang der atypischen Substanz in normal gebaute Kleinhirnrinde.

Fig. 4. Eine atypisch gebaute Stelle aus dem unteren Wurm. Man sieht verstrente Purkinje-Zellen, daneben größere und kleinere Haufen von Körnern. Der übrige Teil der atypisch gebauten Stelle besteht aus mehr homogen aussehender Molekularsubstanz. Das Ganze ist von einem Mantel markhaltiger Fasern umgeben. 

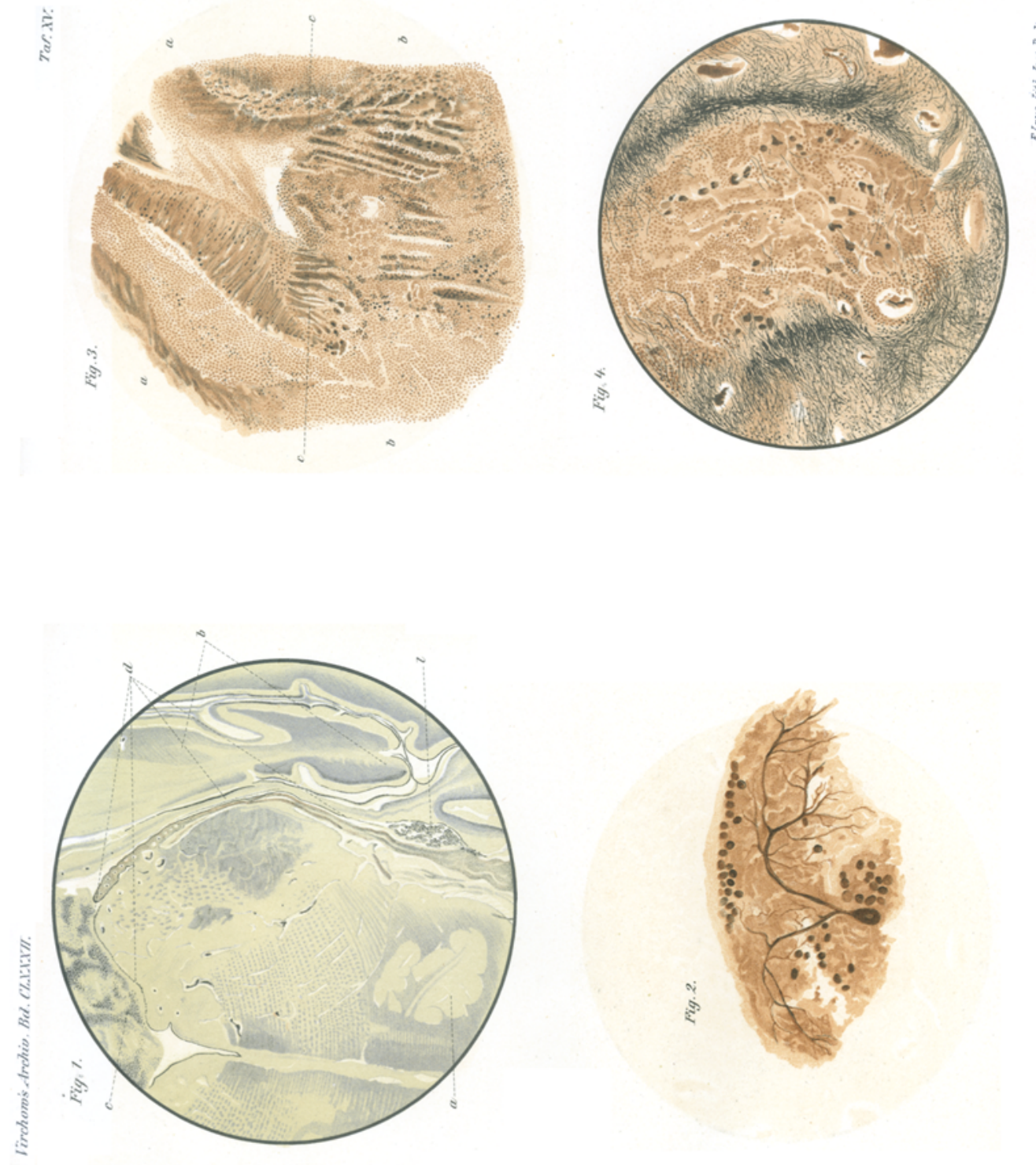